\title{
Methods for Molecular Nanoanalysis
}

\author{
Thomas Schmid, Thomas A. Schmitz, Patrick D. Setz, Boon-Siang Yeo, Weihua Zhang, and \\ Renato Zenobi*
}

\begin{abstract}
This contribution reviews methods based on scanning probe microscopy for molecular analysis and identification on a length scale of 20-200 nm. Aperture scanning near-field optical microscopy (SNOM) was initially developed in our group for chemical analysis and imaging using fluorescence and Raman spectroscopies, with a resolution beyond the optical diffraction limit. In recent years, tip-enhanced Raman spectroscopy was developed into an analytical tool capable of a spatial resolution less than $50 \mathrm{~nm}$ and, using the 'gap' mode of operation, the promise for single molecule sensitivity and even higher spatial resolution. Aperture SNOM with pulsed laser irradiation can be used for nanoscale laser ablation. With an interface to a very sensitive mass spectrometer, this provides a platform for nanoscale mass spectrometry using atmospheric pressure sampling. The preparation of fiber tips for aperture SNOM as well as metallized AFM tips and etched metal wires for apertureless SNOM applications is reviewed. Finally, applications for the chemical analysis of thin molecular films and of biological samples are presented.
\end{abstract}

Keywords: Atmospheric pressure nanoscale laser ablation mass spectrometry ·

Scanning near-field optical microscopy (SNOM) · Tip-enhanced Raman scattering (TERS)

\section{Introduction}

The world around us is primarily molecular. Methods for chemical analysis are therefore designed to reveal the molecular nature of matter, and there are many ways to achieve this goal. Historically, solution-phase chemical methods have been used, for example chemical degradation via ozonolysis or specific detection methods based on biomolecular recognition. More recently, instrumental spectroscopy methods such as nuclear magnetic resonance, infrared spectroscopy, or mass spectrometry have been developed to yield detailed information about the molecular composition of matter. However, most of these methodologies cannot be applied to questions in nanoscience and nanotechnology, because they intrinsically lack spatial resolution.

Techniques with nanoscale lateral resolution, such as standard atomic force microscopy (AFM), scanning tunneling microscopy (STM) and scanning electron microscopy (SEM), typically give very little or no chemi-

\footnotetext{
${ }^{*}$ Correspondence: Prof. Dr. R. Zenobi

Department of Chemistry and Applied Biosciences

ETH Zürich

$\mathrm{CH}-8093$ Zürich

E-Mail: zenobi@org.chem.ethz.ch
}

cal information at all. Some exceptions exist, for example derivatized AFM tips that can be used for chemical recognition. In general, however, only the shape of nanostructures, the surface topography, and local electric properties can be measured, albeit in many cases with excellent spatial resolution. With the advent of nanotechnology and nanoscience, the need for chemical analysis has become urgent. Methods of nanotechnology and nanoscience allow us to control and make use of properties of matter on the nanoscale (length scale well below $1 \mu \mathrm{m}$ in all three dimensions). Nano-objects that are under intense study include subcellular compartments, nanomaterials such as polymer blends, 'molecular machines' and small biological objects, nanoparticles with supposed toxic effects, or components of molecular electronics. There is currently a lack of methods capable of chemical diagnostics and characterization, in particular of the molecular composition, of such nano objects. Fig. 1 illustrates some of the challenges.

\section{Principles of Chemical Analysis by Near-Field Methods}

As a platform for nanoscale chemical analysis, we use scanning near-field optical microscopy (SNOM), the 'optical cousin' of STM and AFM. Standard 'aperture SNOM' works as follows: light from a laser is guided into an optical fiber, whose tip is sharpened and metallized on the outside such that only a very small aperture (diameter $\approx 50$ $\mathrm{nm}$ ) passes some laser light. This nanometer light source can be used to illuminate a spot on a sample that is considerably smaller than the optical diffraction limit, $\sim \lambda / 2$ ( $\lambda=$ wavelength of incident laser light). Using a method developed in our group based on chemical etching (see below), SNOM tips with only $50 \mathrm{~nm}$ diameter and a high optical throughput can be fabricated. In order to obtain a high-resolution optical image of the sample, the SNOM tip is scanned over the sample surface with nanometer accuracy, using piezo elements for motion control.

In principle, the use of SNOM not only allows topographic and optical but also chemical information to be obtained, by optically dispersing the emitted or inelastically scattered light to yield fluorescence or Raman spectra (Fig. 2a). This is a nondestructive way of analyzing surfaces. An alternative is nano ablation using laser pulses with SNOM tips (Fig. 2b). SNOM-laser ablation experiments have also been done in our laboratory already 10 years ago [1][2]. We have also interfaced SNOM-laser ablation with mass spectrometric analysis of the ablation products. The big challenge is the extremely small amount of material ablated (only $c a$. $10^{6}$ molecules per laser shot for a $100 \mathrm{~nm}$ crater). A key paper from our laboratory demonstrated the feasibility of this strategy [3]. In this work, laser ablation was performed at atmospheric pressure, which is advantageous for samples such as tissue sections that cannot withstand the high vacuum 

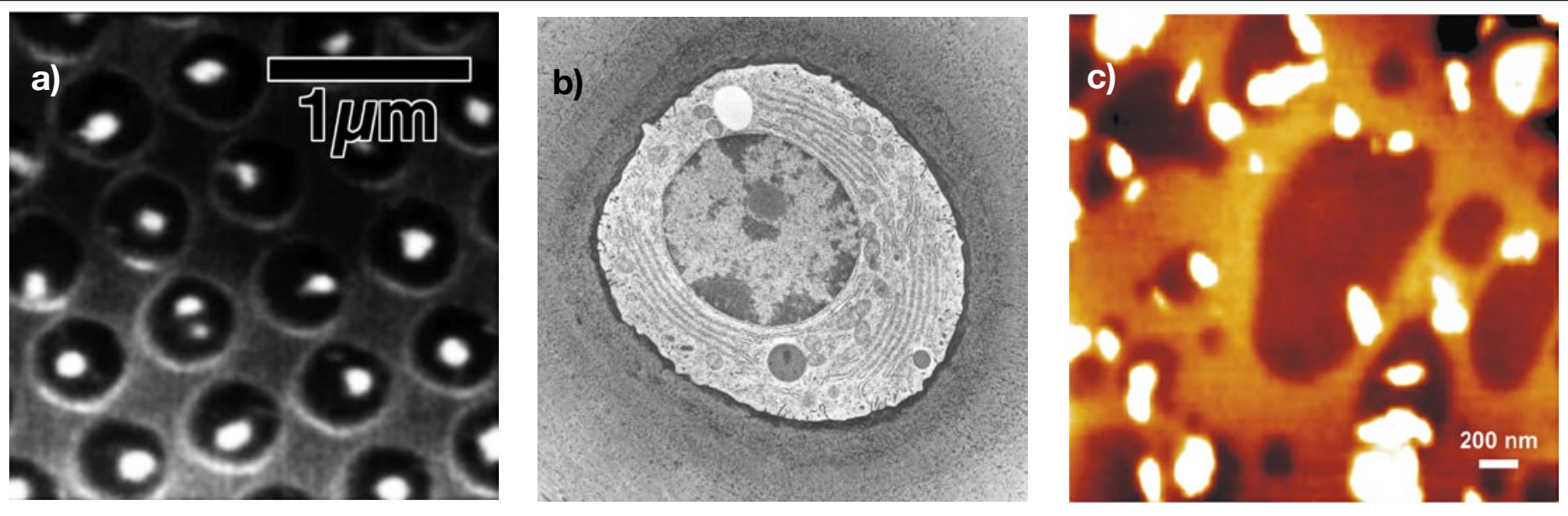

Fig. 1. (a) Challenge \#1: Electron micrograph of a model catalyst (Pd islands on a nanostructured $\mathrm{SiO}_{2}$ substrate formed by annealing). What is the chemical nature of reactants, intermediates, and products present at the active sites of the catalyst? (b) Challenge \#2: Electron micrograph of a chondrocyte. What is the chemical composition of the subcellular compartments? (Courtesy of Dr. Daniel Studer, University of Bern). (c) Challenge \#3: AFM/tapping mode measurement of a microtome thin section of a model rubber blend. What is the chemical identity of the different domains?

conditions typical of mass spectrometers. The laser ablated material was sucked into the vacuum system using a capillary interface, followed by ionization using electron impact and mass analysis using a quadrupole mass spectrometer.

The particular challenge in coupling SNOM with Raman spectroscopy is the fact that Raman scattering is an inherently weak effect. In addition, if Raman scattered light originates from a very small (nanometersized) region, it is normally barely detectable. A way to circumvent this problem is to employ surface-enhanced Raman scattering (SERS), based on local enhancement of the electromagnetic fields (by up to six orders of magnitude), by depositing samples on a rough film of silver. Certain shapes and sizes of silver colloids are able to enhance the Raman scattering of adsorbed sample molecules by more than 12 (!) orders of magnitude. The special approach we have pioneered makes use of an 'apertureless' version of SNOM, where the Raman scattering is amplified by the presence of a metallic nanoparticle brought to the sample by means of an atomic force microscope cantilever tip (Fig. 2c). We call this 'tip-enhanced Raman scattering' or TERS [4]. The advantage of this approach is that the sample can be illuminated using conventional optics, avoiding the comparatively lower transmission of an aperture SNOM tip. Such apertureless nearfield spectroscopy experiments have in the meantime been realized using coated AFM tips or sharpened metal tips. A special geometry that promises particularly high enhancement of the Raman scattered light is shown in Fig. 2d ('gap-mode TERS'). The sample is localized in the gap between a metallic surface and a sharp STM tip. STM feedback is used to bring the tip within a nanometer or less of the surface.

\subsection{Preparation of Aperture SNOM Tips}

\subsubsection{Taper Formation: Tube Etching}

In particular for SNOM-Raman measurements and for atmospheric pressure SNOMMS [3], aperture SNOM tips with a high optical throughput and high damage threshold are necessary. To produce such SNOM probes with aperture dimensions of $100 \mathrm{~nm}$ diam. or less, a highly reproducible and efficient fabrication method called 'tube etching' has been developed and studied in detail in our laboratories (Fig. 3). 'Tube etching' yields tips with excellent quality regarding taper angle and surface smoothness [5-7].

Tube etching is usually done batch-wise (eight fibers simultaneously) with $40 \%$ hydrofluoric acid. An organic overlayer (e.g. iso-octane) is used to protect the fiber mounts from the corrosive HF vapor. The polymer coatings at the tapered end of the fibers are removed by treatment with hot concentrated sulphuric acid. After thoroughly washing in $\mathrm{H}_{2} \mathrm{O}$ and $\mathrm{EtOH}$ to remove traces of decomposed coatings and residual $\mathrm{H}_{2} \mathrm{SO}_{4}$, the tips are finally dried overnight in a desiccator prior to metallization.

\subsubsection{Metallization and Aperture Formation}

To prevent the light from leaking through the tapered side of the tip as well as to create a well-defined aperture, a metal coating is applied onto the tapered region of the fiber.
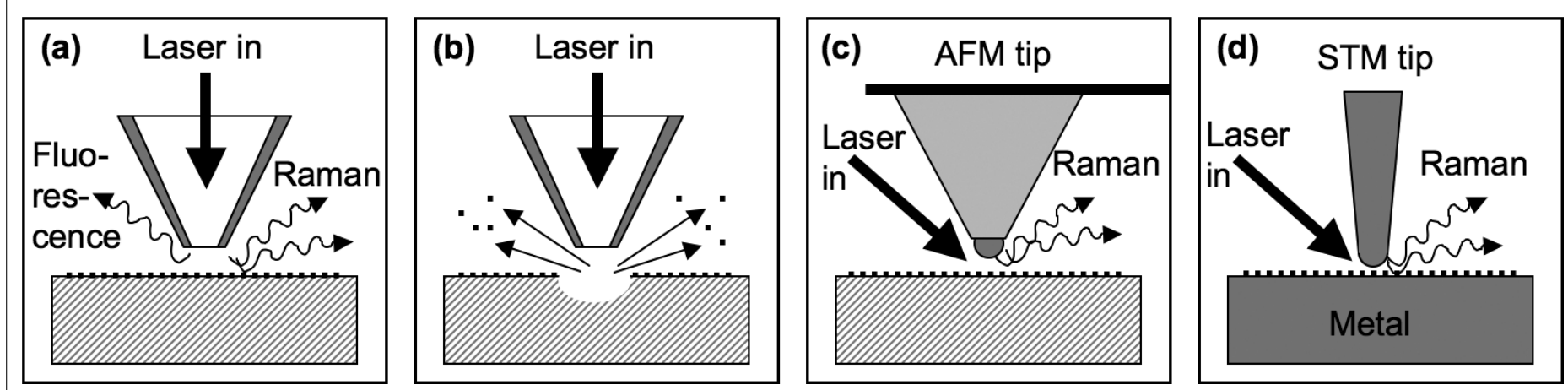

Fig. 2. Different operating modes of near-field spectroscopic methods: (a) aperture SNOM for exciting fluorescence or Raman scattering on a sample surface. (b) Aperture SNOM for pulsed nanoscale laser ablation with subsequent mass spectrometric detection of the ablation products. (c) Apertureless SNOM using a modified AFM tip for tip-enhanced Raman scattering (TERS). (d) Use of a metallic STM tip in very close proximity to the sample surface for gap-mode TERS. 


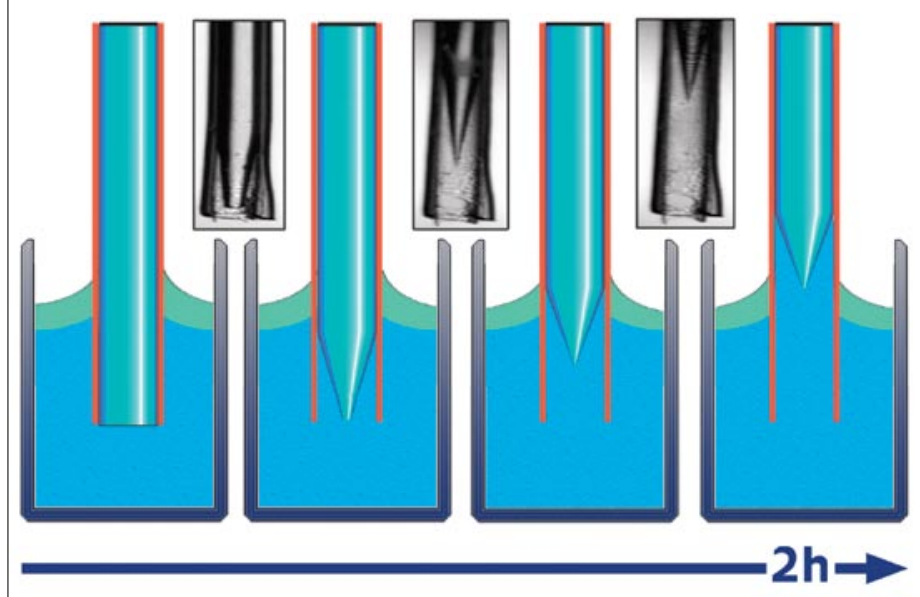

Fig. 3. Schematic of 'tube etching'. The insets show selected video frames acquired during such an etching process (adapted from [5]).

To achieve this, an adhesion layer coating method is often used. In this technique, alternating layers of $\mathrm{Cr}$ and $\mathrm{Al}$ were deposited on the bare tapered glass to increase the stability of the overall metal coating. The resulting sandwich-like coating has a total thickness of $170-180 \mathrm{~nm}$. During metal evaporation the fiber is continuously rotated around its axis, thus yielding a homogeneous metallization around the taper, while at the very end the tip remained uncoated because of a shadowing effect (taper is tilted away from the metal vapor source). Tips produced in this manner showed a superior optical destruction threshold and could withstand up to $170 \mu \mathrm{J}$ of pulsed laser radiation before the coating ruptured. To check for quality of etching and metallization, light from a $\mathrm{HeNe}$ laser is coupled into the cleaved end of the fibers and the tapered end of the tips is then optically inspected with a light microscope for shape and opacity. Only homogeneously coated tips that do not leak any light except at the apex are then further investigated by SEM. Fig. 4 shows SEM images yielding information about the shape of the taper and the size of the aperture.

After inspection and choice of successfully fabricated fiber tips, they are glued onto tuning forks with fast hardening glue and are ready for use as SNOM probes.

\subsection{Preparation of Apertureless Tips}

Shape and material of the tip have direct effect on the degree of TERS enhancement, and consequently, the signal intensity and $\mathrm{S} / \mathrm{N}$ ratio. Furthermore, the lateral resolution of the technique is directly related to the dimensions of the tip end. Its fabrication procedure is thus of great importance for the performance of TERS as an analytical tool. The type of tip used is closely related to the method to control the tip-sample distance. In our group, two types of SPM techniques, AFM and STM, are currently used for TERS experiments.
The AFM is the most universal, versatile and robust method for surface analysis, with the advantage of being able to obtain complementary data such as friction force, electric force, magnetic force, or phase images simultaneously with the topography image. Two types of methods have been used to metallize AFM tips: the widely used vapor deposition method [4][8] and chemical deposition (Ag mirror reaction) [9]. In our group, AFM tips for TERS are usually prepared by vapor coating of commercially available AFM tips with Ag layers with a thickness of a few tens of nanometers. Typical examples are shown in Fig. 5a-d. AFMTERS experiments are described in Section $4.1[10]$

Compared to AFM, the fabrication of the STM tip for gap-mode TERS is easier and cheaper. The main disadvantage is that STM-based TERS requires conductive samples, which limits its application. In the area of STM, people have accumulated a lot of knowledge for tip fabrication [11] The best Raman enhancement to date has been reported from gap-mode TERS studies with $\mathrm{Au}$ tips [12][13]. The imaginary part of the permittivity of $\mathrm{Ag}$ in the visible range is much smaller than that of $\mathrm{Au}$, which leads to a higher field enhancement. This has been observed in our experiment, where by use of Ag tips, single molecule sensitivity is attainable (see Section 4.2). Our Ag tips

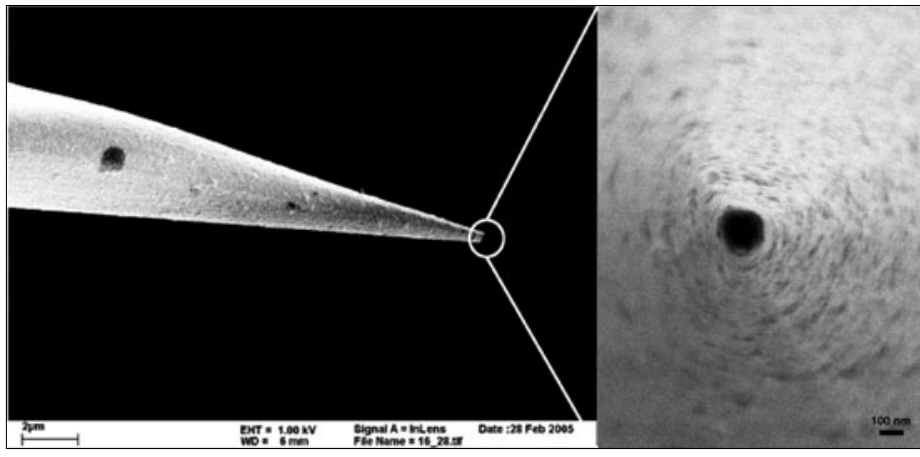

Fig. 4. SEM image of a tube-etched and metallized tip. The left image illustrates the smoothness of the tapering, while in the right image a magnification of the same tip is shown (top view) in order to investigate the size and shape of the aperture. Scale bar left image: $2 \mu \mathrm{m}$; right image: $100 \mathrm{~nm}$ with rubidium. It took over $10 \mathrm{~h}$ to complete for gap-mode TERS are prepared with the etching method developed by Iwami et al. $50 \mathrm{~nm}$ can be achieved reproducibly (see Fig. 5e).

We believe that the ability to reproducbly fabricate tips that exhibit consistently TERS enhancement with a particula widespread application of TERS for na-

\section{Aperture SNOM in Chemical Analysis}

\subsection{Fluorescence} dyes allowed imaging of single molecules bocyanine dye spread on a cover slip that as previously spin coated with a $30 \mathrm{~nm}$ Pof polymethylmethacrylate of 23 molecules $/ \mu \mathrm{m}^{2}$. The SNOM was used to image individual dye molecules. In the same work the orientation of each molecule was determined. The results were used to than other spectroscopic methods, because

\subsection{Raman/SERS}

Raman spectroscopy is a well-estab molecular species, material phases, and stress through the analysis of the vibrational states. It is non-destructive and it does not impose strict requirements on the preparasolutions. The first near-field Raman image was obtained in 1995 by Jahncke et al. [16] on potassium titanyl phosphate doped 

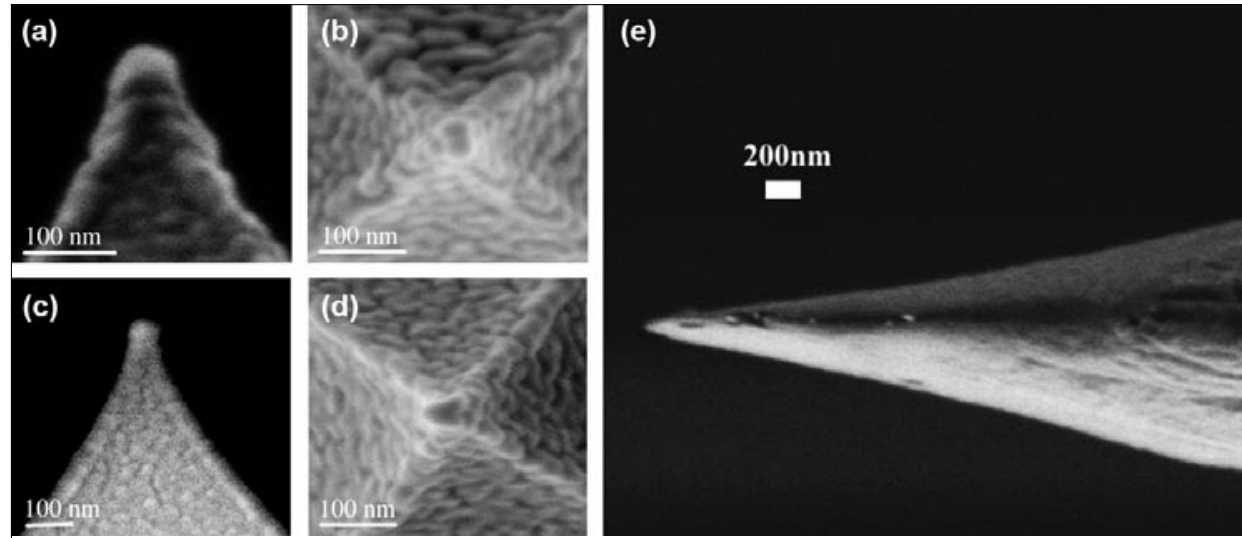

Fig. 5. SEM images of typical TERS tips: side-views (a, c) and corresponding top-views (b, d) of Agcoated AFM tips and side-view of an electrochemically etched Ag wire for gap-mode TERS (e).

the scan but the spectroscopic analysis of the Raman light could clearly identify the doped zones in the crystal. Nevertheless, the small scattering cross section and the luminescence background can obscure the weak Raman bands of many samples. This limitation can be overcome by exploiting the surface enhanced Raman scattering (SERS) effect: molecules adsorbed on specially prepared metal substrates show an enhancement of their Raman intensity of several orders of magnitude. In our laboratory, SERS chemical imaging was demonstrated [17] using brilliant cresyl blue-labeled DNA deposited on a substrate covered with silver-coated polytetrafluoroethylene (PTFE) nanospheres. A resolution of $100 \times 100 \mathrm{~nm}^{2}$ was achieved.

\subsection{SNOM-Laser Ablation MS}

Chemical analysis by mass spectrometry (MS) is one of the most significant analytical techniques for the identification of unknown compounds and quantification of known substances in complex matrices. Laser ablation MS is conventionally used for the spatially resolved analysis of surfaces but resolution is limited to $5-25 \mu \mathrm{m}$ due to practical optical limitations and ultimately due to the diffraction limit. The only alternative MS method, which routinely achieves 50-100 nm spatial resolution using a focused ion beam, is secondary ion mass spectrometry (SIMS). Unfortunately, this method is limited to analysis under ultra-high vacuum conditions and only suitable for small molecules since it is a relatively 'hard' ionization method. Due to these limitations, there is no analytical method available today for the nano-analysis of organic compounds with MS, especially with regards to biological samples (i.e. analysis of metabolites in living cells or tissue).

In 2001, our group showed the feasibility of combining SNOM-based laser ablation with mass spectrometry at atmospheric pressure for the first time [3]. To overcome previous instrumental limitations, we have developed in the meantime a SNOM-MS instrument with a highly sensitive ion trap time-of-flight (IT-TOF) mass analyzer built specially for this application [18]. A schematic is shown in Fig. 6.

A SNOM fiber tip with an optical aperture of 50-150 $\mathrm{nm}$ is used as a light source for laser ablation on the nanometer scale and combined with the IT-TOF MS by a transfer ('suction') capillary. While the tip is kept in shear-force feedback some 5-15 nm above the surface of the sample, a topographic image of the surface can be acquired. At any desired location, the UV laser coupled into the fiber is switched on for ablation; a spatial resolution of $70 \mathrm{~nm}$ (FWHM) has been achieved. The ablated capillary, transferred into the ion trap and ionized therein. The IT serves as a storage and accumulation/pre-concentration device and is pulsed into a time-of-flight (TOF) mass analyzer. This setup will allow chemical analysis with nanometer spatial resolution at atmospheric pressure for numerous applications in materials and life sciences. analyte is then 'sucked' into the sampling right part: IT-TOF mass spectrometer)

\section{TERS in Chemical Analysis}

\subsection{AFM-TERS}

The aim of this project is the development of an analytical tool for chemical analysis of sample surfaces with nanoscale spatial resolution. The ability to perform such measurements, especially if it can be done in situ and nondestructively, is of growing importance. In 2000, our group was the first to experimentally demonstrate TERS [4]. By harvesting the spatial resolution and chemical specificity afforded by scanning probe microscopy (SPM) and Raman spectroscopy, respectively, the surface compositions of brilliant cresyl blue (BCB) and $\mathrm{C}_{60}$ thin films have been unambiguously fingerprinted with a 50-nm spatial resolution.

A large near-field enhancement of the Raman signals is essential for performing rapid TERS imaging. Unfortunately, the experimental Raman enhancements have been lower than theoretical predictions. This has hampered the development of TERS to become a robust and commercially useful technique. We have been actively involved in the fabrication and design of various TERS probes based on AFM tips, which are intimately connected to Raman enhancements possible in an experiment. A contributing factor has been identified as being caused by the effect of the AFM tip material on the wavelength of the surface plasmon resonance $\left(\lambda_{\mathrm{SPR}}\right)$ of the attached Ag particles [10]. In general, the $\lambda_{\mathrm{SPR}}$ of metal particles is known to increase with higher refractive index (n) of the underlying substrate. Using a 488-nm excitation laser, we have successfully demonstrated the circumvention of this problem by using AFM tips made of $\mathrm{SiO}_{2}$ $(\mathrm{n}=1.5)$, which has a lower $\mathrm{n}$ compared to $\mathrm{SiN}(\mathrm{n}=2.05)$ or $\mathrm{Si}(\mathrm{n}=4.4)$. This is shown in Fig. 7 where the Raman signals of a BCB thin film is better enhanced with a Ag-coated $\mathrm{SiO}_{2}$ tip, as compared to the best possible

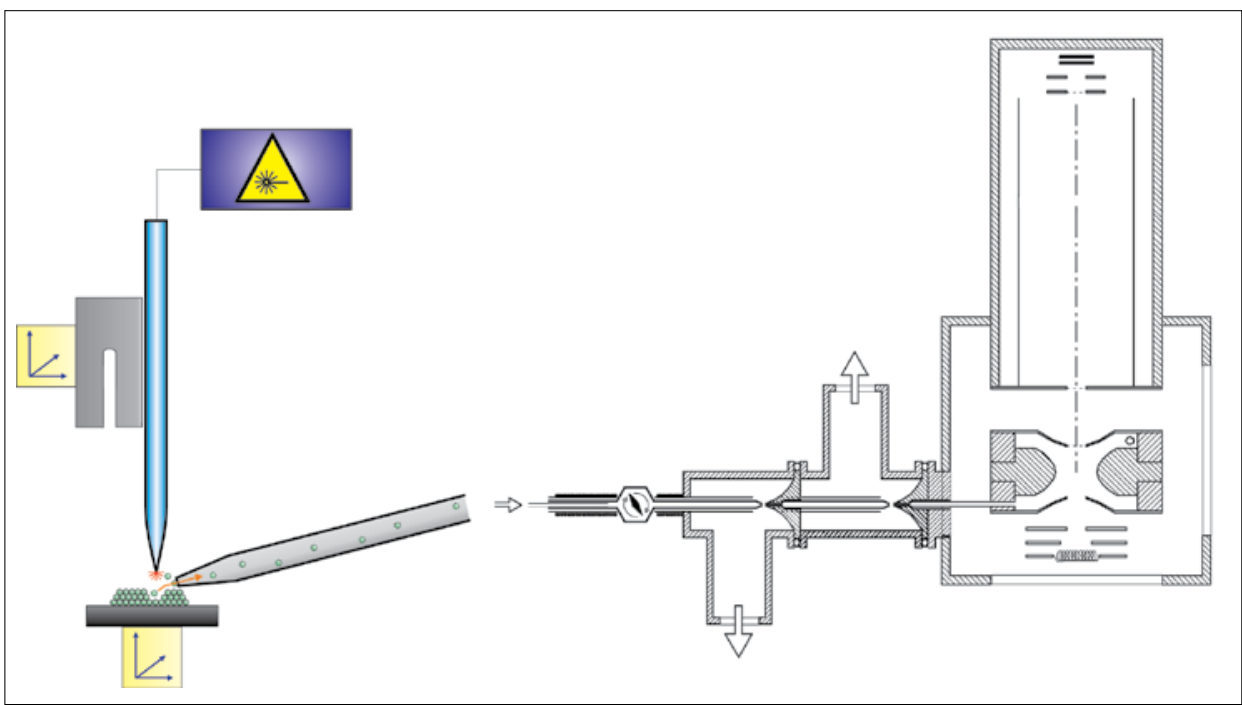

Fig. 6. Instrumental setup of the SNOM-MS setup (left part: SNOM with laser coupled into the fiber, 


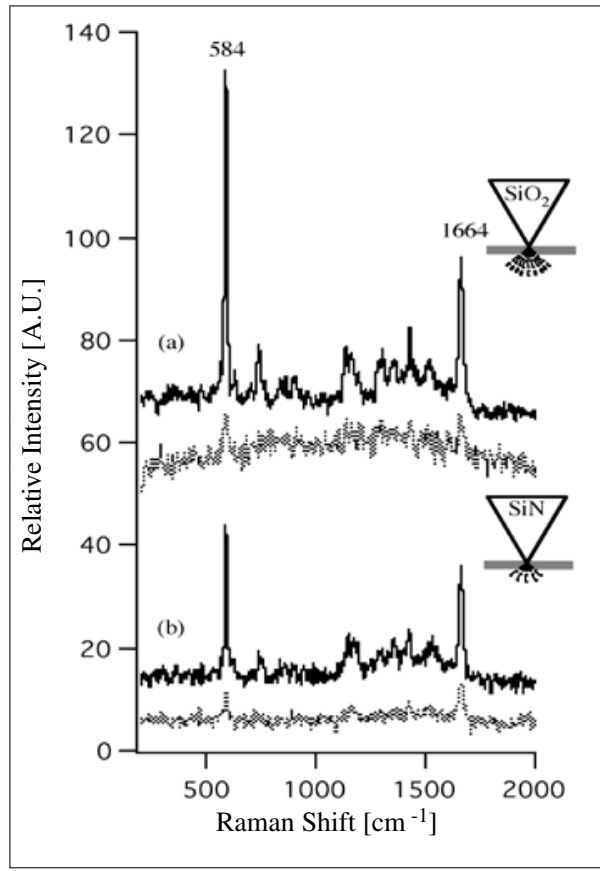

Fig. 7. Raman spectra of brilliant cresyl blue acquired with tip in contact (dark trace) and retracted (dotted trace). TER spectrum is collected with a (a) Ag coated $\mathrm{SiO}_{2}$ tip and (b) $\mathrm{Ag}$ coated SiN tip (adapted from [10]).

signal enhancement obtained by us for similarly coated $\mathrm{SiN}$ tips.

\subsection{Gap-mode TERS}

Besides using a single nanostructure like the TERS setup described above, other geometries can be also used for TERS. Electromagnetic theory predicts a strongly localized surface plasmon mode in the nano-junction between a flat metal surface and a metal sphere [19], called 'gap-mode'. For this TERS scheme, enhancement factors $>10^{7}$ can be expected. Simulations show that the electric field strength can be also enhanced by $10^{2}$ times at resonant condition [20][21]. This structure is naturally related to the STM, which uses a sharp metal tip and a flat conductive substrate. The first successful gap-mode TERS setup was realized using an STM and electrochemically etched Au tips. A $10^{6}$-fold enhancement was observed [12]. In our lab, Ag tips are used instead of Au tips, because the imaginary part of permittivity of $\mathrm{Ag}$ is much smaller than $\mathrm{Au}$ in visible light range [22] and thus the enhancement should, in theory, be even larger. This has indeed been observed in our experiments.

A sub-monolayer of $\mathrm{BCB}$ was used to demonstrate the power of this gap-mode TERS. The samples were made with following steps:

i) An Au substrate was produced by vapor coating a 150-nm Au layer onto a freshly cleaved mica substrate.

ii) The Au film was annealed using a butane micro-torch. iii) A $10^{-5} \mathrm{M}$ BCB solution was spin-coated onto the substrate.

Fig. 8 shows two spectra recorded from this sample. When the tip is far away from the sample surface, no Raman signal can be observed; after the tip approached to the tunneling range where the tip is typically within $1 \mathrm{~nm}$ of the sample surface, a strong signal from BCB molecules appears.

The TERS cross-section can be estimated from the sample coverage (15 molecules per $\left.100 \mathrm{~nm}^{2}\right)$, the power $(0.5 \mathrm{~mW})$ and the area of the focus $\left(5 \mu \mathrm{m}^{2}\right)$ of the illumination laser. Besides these parameters, the quantum efficiency of the CCD $(80 \%)$ and the efficiency of the optical system also need to be taken into account $(\sim 1 \%)$. The TERS cross-section of the BCB molecules is estimated to be at least $4 \times 10^{-18} \mathrm{~cm}^{2}$. With such a large cross-section, single molecule TERS is within reach. Considering the $\mathrm{S} / \mathrm{N}$ ratio, $10^{-18} \mathrm{~cm}^{2}$ is the normal threshold for single molecule detection in fluorescence microscopy [23]. Though it is smaller than the absorption cross-section of laser dye molecules, it is still detectable. Because an integrating detector (CCD camera) is used in TERS experiments, a simple increase of the collection time will yield a better $\mathrm{S} / \mathrm{N}$ ratio. However, more research will be necessary to unambiguously prove single-molecule sensitivity of gap-mode TERS. Key difficulties include 'bleaching' phenomena due to photodecomposition or photodesorption triggered by the intense field under the enhancing tip, the understanding of spectroscopic fluctuation and 'blinking' phenomena in Raman scattering, and the production of sufficiently well characterized submonolayer films for single molecule studies.

With a sensitivity at the single molecule level and the combination with STM, gapmode TERS should aid researchers to understand chemical reactions on metal surfaces at the molecular level. Gap-mode TERS can also help to unravel the mechanism of SERS [24], which is still not completely understood. The main problems are that the SERS active substrate is not a well-defined system and that there is no method to determine how and where the molecules reside on a SERS substrate. In contrast, gap-mode TERS is a

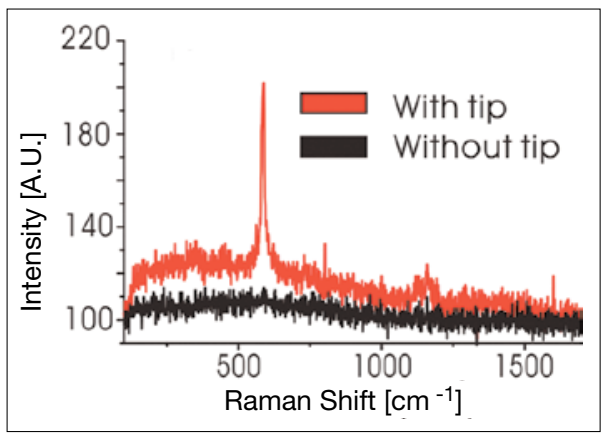

Fig. 8. Comparison of the tip-enhanced Raman spectrum and the far-field Raman fairly well-defined system; it can be done on single crystal surfaces. Furthermore, where and how molecules are bound onto a surface can in principle be determined by STM. The comparison between this information and the Raman spectrum will allow direct insight into the details of the enhancement mechanisms.

\subsection{Analysis of Biological Nanostructures}

Attachment of bacterial cells to solidaqueous interfaces and biofilm formation are processes that are dominated by nanometer-sized structures. Bacteria can reversibly attach to solid surfaces by means of nanometer-sized cell-surface structures, i.e. pili and flagella. Immobilized bacteria produce so-called extracellular polymer substances (EPS), which lead to irreversible fixation of bacterial microcolonies and formation of biofilms. The EPS matrix of a biofilm is composed of different polysaccharides, proteins, humic substances, and other biopolymers, which can range in size from nanometers up to a few micrometers. Biofilms are the predominant life form of bacteria in nature and play important roles in medicine, industry, and biotechnology [25-28]. For improvement of biocides for biofilm removal or process optimization in wastewater treatment plants, a detailed knowledge of structure, chemical composition, and interactions at the nanometer scale is necessary [29].

Confocal laser scanning microscopy (CLSM) is widely used to investigate bacteria and biofilms. Besides two- and threedimensional imaging, this technique allows chemical characterization of different parts of the sample by spectroscopic methods, such as fluorescence and Raman spectroscopy. A drawback of CLSM is the restriction of the spatial resolution by the diffraction limit. Therefore, CLSM is mostly applied to the micrometer scale and is not capable of imaging objects with a size of less than a few hundreds of nanometers. On the other hand, scanning probe microscopy techniques, such as AFM, provide imaging of nanometersized biological structures but no information on their chemical composition. In our group, a combined setup was developed, which allows the investigation of the same part of a sample by CLSM, AFM and Raman spectroscopy [30].

First applications of this setup to EPS polysaccharides, bacteria, and biofilms revealed its potential in this field and in the investigation of biological nanostructures in general. Fig. 9b shows a CLSM image of biofilm-forming river water bacteria attached to a glass slide. The false color representation (blue to red) is based on the light intensity backscattered by the sample after 488-nm laser excitation. Tapping mode AFM images of the same part of the sample reveal the sub-micrometer spatial resolution of this 
technique (see Fig. 9d and 9f). In Fig. 9d, different colors reflect different height values, i.e. the topography of the sample. In the first order derivative (i.e. the gradient) of the topography image, even small changes in topography can be clearly identified (see Fig. 9f). In other AFM measurements with higher magnification, even pili, flagella, and other structures on the cell surface with a thickness of a few nanometers were clearly visible. For enhancement of Raman signals from nanostructures, the sample was coated with 40-nm silver colloids, which are clearly visible in the AFM image, but lead only to a weak diffraction pattern with larger size in CLSM due to the diffraction limit. By focusing the laser on silver clusters, enhanced Raman spectra could be measured, which represent a fingerprint of the molecular composition of the adsorbates on the $\mathrm{Ag}$ particles. As can be seen, on a blank part of the glass slide, no signals were achieved (Fig. 9a), whereas the illumination of $\mathrm{Ag}$ clusters, which are in contact with two parts of the biofilm aggregate led to two different Raman spectra (see Fig. 9c and 9e). In other experiments with an EPS polysaccharide, the enhancing particle was brought close to the structures of interest in form of a Agcoated and TERS-active AFM tip.

The aim of further experiments is to image and discriminate different biopolymers (e.g. polysaccharides and proteins) in hydrogels, biofilms, and other biological matrices with nanometer scale spatial resolution. Besides analysis of the internal structure of biofilms, this technique can be an interesting tool for the investigation of sub-cellular structures or cell surfaces and the elucidation of transport pathways of nanoparticles in biological tissues, which are under discussion as potential drug delivery systems.

\section{Conclusions}

Near-field fluorescence spectroscopy, near-field Raman spectroscopy, and nearfield laser ablation mass spectrometry are maturing as powerful analytical methods for the nano world. In the absence of natural fluorophores, fluorescence detection depends on chemical labeling. Raman spectroscopy is a very attractive, nondestructive and label-free method for identification and analysis. In the near field, it can be performed both by aperture and apertureless SNOM methods. The latter has the potential for excellent sensitivity - reaching single molecule detectability - and very high spatial resolution. Mass spectrometric analysis of SNOM-laser ablation products is predicted to become a powerful method of chemical analysis and imaging as well. Although it is destructive, this disadvantage is easily outweighed by the very high
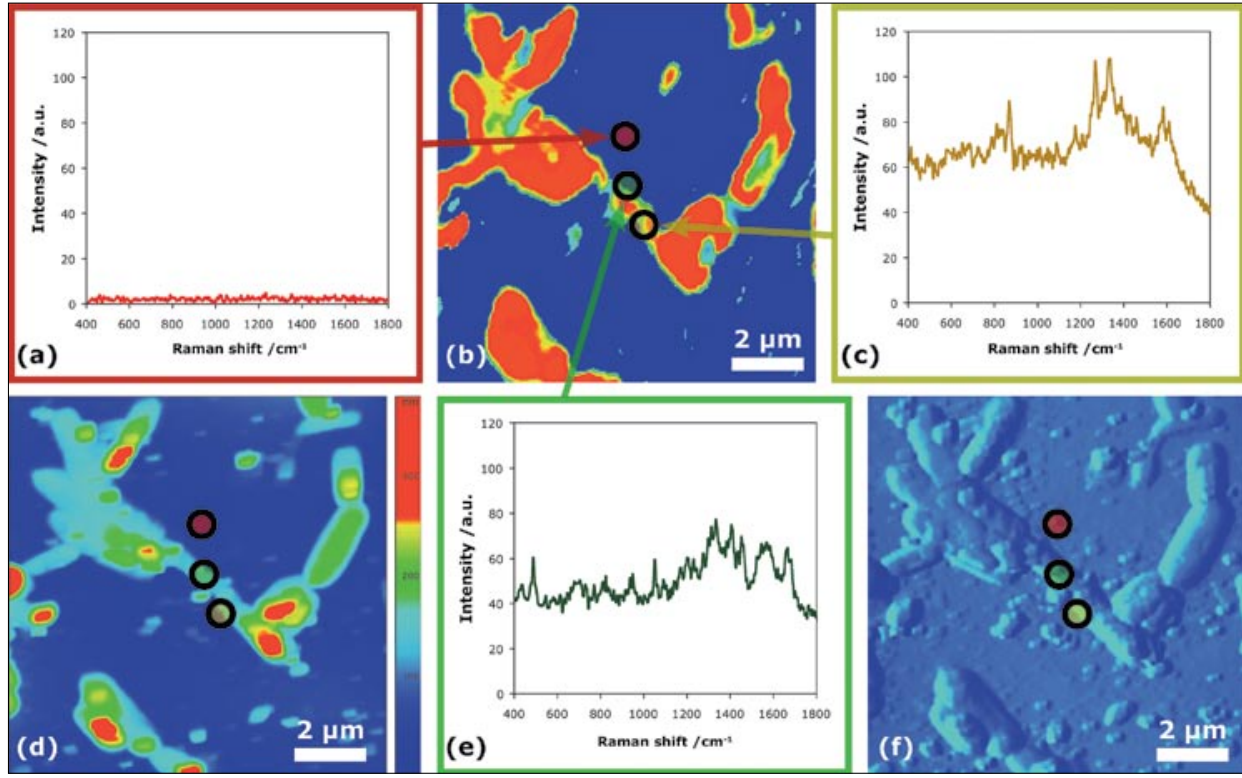

Fig. 9. Imaging and chemical analysis of a bacterial aggregate by CLSM, AFM, and Raman spectroscopy: CLSM image (b), AFM topography (d), AFM topography gradient (f), and Raman spectra of three different parts of the sample $(a, c, e)$.

chemical information content available from mass spectrometry.

\section{Acknowledgments}

The authors would like to express their gratitude to Dr. Frank Krumeich, ETH Zürich, for the SEM measurements as well as Heinz Benz and Kurt Baumgartner, ETH Zürich, for technical support. Financial support for our work from the Deutsche Forschungsgemeinschaft (to Thomas Schmid), the ETH Zürich, and the Gebert Rüf Stiftung (grant no. P-085/03) is greatly appreciated.

Received: September 4, 2006

[1] D. Zeisel, B. Dutoit, V. Deckert, T. Roth, R. Zenobi, Anal. Chem. 1997, 69, 749.

[2] D. Zeisel, S. Nettesheim, B. Dutoit, R Zenobi, Appl. Phys. Lett. 1996, 68, 2491.

[3] R. Stöckle, P. Setz, V. Deckert, T. Lippert, A. Wokaun, R. Zenobi, Anal. Chem. 2001, 73, 1399.

[4] R.M. Stöckle, Y.D. Suh, V. Deckert, R Zenobi, Chem. Phys. Lett. 2000, 318, 131.

[5] R.M. Stöckle, PhD Thesis ETH Zürich No. 13797, 2000.

[6] R. Stöckle, C. Fokas, V. Deckert, R. Zenobi, B. Sick, B. Hecht, U.P. Wild, Appl. Phys. Lett. 1999, 75, 160.

[7] R.M. Stöckle, N. Schaller, V. Deckert, C. Fokas, R. Zenobi, J. Microsc.-Oxford 1999, 194, 378.

[8] N. Hayazawa, Y. Inouye, Z. Sekkat, S. Kawata, Opt. Commun. 2000, 183, 333.

[9] J.J. Wang, Y. Saito, D.N. Batchelder, J. Kirkham, C. Robinson, D.A. Smith, Appl. Phys. Lett. 2005, 86, 263111.

[10] B.S. Yeo, W. Zhang, C. Vannier, R. Zenobi, Appl. Spectrosc. 2006, 60, 1142.

[11] A.J. Melmed, J. Vac. Sci. Technol. B 1990 , 9, 601 .

[12] B. Pettinger, B. Ren, G. Picardi, R. Schuster, G. Ertl, Phys. Rev. Lett. 2004, 92, 096101.
[13] B. Ren, G. Picardi, B. Pettinger, R. Schuster, G. Ertl, Angew. Chem. Int. Ed. 2005, 44, 139.

[14] M. Iwami, Y. Uehara, S. Ushioda, Rev. Sci. Instrum. 1998, 69, 4010.

[15] E. Betzig, R. J. Chichester, Science 1993 $262,1422$.

[16] C.L. Jahncke, M.A. Paesler, H.D. Hallen, Appl. Phys. Lett. 1995, 67, 2483.

[17] V. Deckert, D. Zeisel, R. Zenobi, T. VoDinh, Anal. Chem. 1998, 70, 2646.

[18] P.D. Setz, T.A. Schmitz, R. Zenobi, Rev. Sci. Instrum. 2006, 77, 024101

[19] P.K. Aravind, H. Metiu, Surf. Sci. 1983, 124, 506.

[20] R.M. Roth, N.C. Panoiu, M.M. Adams, R.M. Osgood, C.C. Neacsu, M.B. Raschke, Opt. Express 2006, 14, 2921.

[21] I. Notingherr, A. Elfick, J. Phys. Chem. B 2005, 109, 15699 .

[22] P.B. Johnson, R.W. Christy, Phys. Rev. B 1972, 6, 4370 .

[23] B. Pettinger, G. Picardi, R. Schuster, G Ertl, Single Mol. 2002, 3, 285.

[24] D.L. Jeanmaire, R.P. Duyne, J. Electroanal. Chem. 1977, 84, 1 .

[25] 'Biofilms in Wastewater Treatment - An Interdisciplinary Approach', Eds. P.L. Bishop, P.A. Wilderer, S. Wuertz, IWA Publishing, London, 2003.

[26] H.C. Flemming, Appl. Microbiol. Biotech. 2002, 59, 629 .

[27] H.C. Flemming, J. Wingender, Biologie in unserer Zeit 2001, 31, 169.

[28] 'Biofilms in Medicine, Industry and Environmental Biotechnology - Characteristics, Analysis and Control', Eds. V. O'Flaherty, A.P. Moran, P. Stoodley, T. Mahony, P. Lens, IWA Publishing, London, 2003.

[29] I.W. Sutherland, Trends in Microbiology 2001, 9, 222.

[30] C. Vannier, B.S. Yeo, J. Melanson, R. Zenobi, Rev. Sci. Instrum. 2006, 77, 023104. 